\title{
Les livres
}

\section{Histoire naturelle et artificielle de la procréation}

\section{J. GonZales}

Bordas, Cultures, Paris, 1996, 400 p, 345 F.

Ce fort volume illustré est destiné à un large public.

J.Gonzales est Professeur de Biologie du Développement et de la Reproduction à l'Université Paris VI, biologiste à l'Hôpital Pitié-Salpétrière et responsable du Centre de documentaiton d'histoire de la médecine de Paris VI. C'est dire que l'auteur est pleinement concerné par son sujet.

L'ouvrage retrace l'histoire des représentations, des idées, des connaissances et des techniques de l'époque préhistorique à nos jours.

A l'époque préhistorique correspond l'ère des substances : l'eau, le sang, le lait sont des symboles forts de vie et leurs représentations sont souvent l'objet des dessins rupestres.

A partir du néolithique, l'écriture formalise une pensée qui relie la procréation à des concepts philosophiques touchant à l'origine du monde et à la genèse des premiers hommes.

A partir du VIe siècle avant J.C., à la suite d'Hippocrate, la génération de l'homme est rapportée à l'effet de semences.
A la Renaissance, l'anatomie des organes génitaux masculins et féminins se précise, mais leur fonctionnement reste mystérieux. L'œuf est reconnu à l'origine de l'individu ; celui-ci est-il préexistant, en miniature, dans l'œuf (préformisme) ou se constitue-t-il à partir d'une substance amorphe (épigenèse)?

A la fin du XVIIe siècle commence la bataille des ovistes qui pensent que la conception d'un enfant ne relève que de la femme, dont les ovaires pondent des oufs, et les animalculistes qui attribuent toute la responsabilité à l'homme puisque la tête de chaque spermatozoïde contient un petit embryon.

$\mathrm{Au}$ XIXe siècle sont reconnues la vraie nature de l'ovule et des spermatozoïdes. La fécondation est observée pour la première fois en 1875 ; les chromosnmes sont découverts en 1880 .

$\mathrm{Au} \mathrm{XXe}$ siècle est levé le mystère de la physiologie des appareils génitaux et éclate la double révolution de la pilule et de la fécondation in vitro.

L'auteur s'est efforcé de relater l'évolution des connaissances et surtout des représentations de la procréation, dans le contexte plus général de l'évolution de la philosophie et de la pensée scientifique. Très documenté, l'ouvrage, de lecture agréable, contient quelque 136 illustrations dont le propos, très heureux, est avant tout de montrer les représentations et fantasmes de tout temps liés à la procréation. 


\section{Questions d'hommes}

\section{J. Belaisch, A. DE Kervasdoue}

Editions Odile Jacob, Guide, Paris, 1996, 797 pages, $195 \mathrm{~F}$.

Jean Belaïsch andrologue et gynécologue, est l'un des fondateurs de la SALF. Anne de Kervasdoué est gynécologue.

Les auteurs annoncent clairement leur propos en introduction : "C'est un guide qui s'adresse à un public large : les hommes pourront le consulter trouvant selon les âges de la vie (adolescent, jeune adulte ou homme mûr) un éclairage aux interrogations qui les préoccupent. Mais les femmes seront aussi des lectrices attentives de ces pages au long desquelles elles pourront apprendre à mieux comprendre le petit garçon, depuis son plus jeune âge jusqu'à l'âge adulte, ainsi que l'homme à travers son parcours sur le chemin de la vie».

L'ambitieuse table des matières nous annonce que nous avons en main un véritable traité d'Andrologie pour large public. Qu'on en juge par le résumé du sommaire :

- Le Corps au masculin : l'appareil génital et son anatomie ; un fonctionnement continu ; une hormone puissante : la testostérone ; une sexualité tournée vers l'extérieur.
- Les âges de la vie : le petit garçon de 0 à 9 ans ; l'éveil de la virilité de 10 à 25 ans ; le jeune adulte de 25 à 40 ans ; la force de l'âge : l'homme de 40 à 60 ans ; la maturité.

- La médecine au service de l'homme : l'examen andrologique. Les maladies de la fonction sexuelle : les troubles de l'éjaculation ; les troubles de l'érection ; la stérilité masculine ; les MST ; le sida ; les maladies de la verge; les maladies des bourses et des testicules; les maladies de la prostate; les maladies du sein ; les comportements sexuels déviants; les autres maladies; la testostérone.

Le lecteur n'est pas déçu, tout y est. La présentation est à mi-chemin entre l'ouvrage de médecine et le guide pratique de l'usager (figurent même les «adresses utiles»). La lecture est facile et agréable. Des dessins de Claire Brétecher, d'un humour policé, viennent égayer discrètement un texte dont la volonté didactique freine le lyrisme spontané de Jean Belaïsch.

L'ouvrage est sérieux, complet, utile. Nous ne lui connaissons pas d'équivalent et nous le croyons promis à un large succès. 